\title{
Effects of exercise therapy on knee joint function and synovial fluid cytokine levels in patients with knee osteoarthritis
}

\author{
SHAO-LAN ZHANG ${ }^{1}$, HONG-QI LIU ${ }^{2}$, XIAO-ZU XU ${ }^{2}$, JUAN ZHI ${ }^{1}$, JIAO-JIAO GENG ${ }^{1}$ and JIN CHEN $^{1}$ \\ ${ }^{1}$ Department of Medical Technology, Yancheng Health Vocational and Technical College, Yancheng 224006; \\ ${ }^{2}$ Department of Oncology, The First People's Hospital of Yancheng City, \\ Yancheng 224005, Jiangsu, P.R. China
}

Received April 24, 2012; Accepted September 10, 2012

DOI: $10.3892 / \mathrm{mmr} .2012 .1168$

\begin{abstract}
The aims of this study were to observe the effect of exercise therapy on the function of the knee joint and the levels of cytokines and cytokine-related genes, specifically tumor necrosis factor- $\alpha(\mathrm{TNF}-\alpha)$, high sensitivity C-reactive protein (hs-CRP) and matrix metalloproteinase-3 (MMP-3), in the synovial joints of patients with knee osteoarthritis (KOA) and to explore its mechanism of action. A total of $100 \mathrm{KOA}$ patients were divided into a treatment group $(n=50)$ and a control group $(n=50)$ according to the order of admission. The patients in the treatment group were treated with diclofenac sodium combined with exercise therapy and the patients in the control group were treated with diclofenac sodium only. The function of the knee joint and the therapeutic efficacy was evaluated and the TNF- $\alpha$, hs-CRP and MMP-3 levels in the synovial fluid were measured following 4 weeks of treatment. The results revealed that the knee joint index score and the TNF- $\alpha$, hs-CRP and MMP-3 levels in the synovial fluid decreased significantly in the KOA patients of the two groups following treatment $(\mathrm{P}<0.05)$. Compared with the control group, the knee joint index score and the TNF- $\alpha$, hs-CRP and MMP-3 levels in the synovial joints were lower and the therapeutic efficacy was increased in the patients of the treatment group $(\mathrm{P}<0.05)$. In brief, exercise therapy may decrease cytokine and cytokine-related gene levels in the synovial fluid and inhibit inflammatory factor-mediated cartilage degradation in KOA patients, thus, effectively improving the clinical symptoms of KOA.
\end{abstract}

Correspondence to: Professor Shao-Lan Zhang, Department of Medical Technology, Yancheng Health Vocational and Technical College, 263 Jiefangnan Road, Yancheng 224006, Jiangsu Province, P.R. China

E-mail: shaolan2004@126.com

Key words: exercise therapy, knee osteoarthritis, knee joint function, TNF- $\alpha$, hs-CRP, MMP-3

\section{Introduction}

Knee osteoarthritis (KOA), also known as degenerative arthropathy and hypertrophic arthritis, is one of the most common degenerative diseases. Its basic pathological changes include degeneration, loss of articular cartilage and osteophyte formation in the articular cartilage and the lower border of the cartilage that manifests as joint pain, tenderness, stiffness, joint swelling, limited mobility and joint deformities, usually involving the finger joints, knees, spine and hip (1). Kinesitherapy may provide therapeutic effects in the treatment of KOA (2-3). The current study used kinesitherapy to treat patients with KOA and explored its mechanism of action by concurrently measuring knee function and the levels of tumor necrosis factor- $\alpha$ (TNF- $\alpha)$, high sensitivity C-reactive protein (hs-CRP) and matrix metalloproteinase 3 (MMP-3) in the synovial fluid.

\section{Materials and methods}

\section{Clinical design.}

Study sample. Patients ( $\mathrm{n}=100,38$ male and 62 female, aged 41-63 years) were diagnosed with KOA in the First People's Hospital of Yancheng City from January 2010 to December 2011. All patients provided their informed consent. The patients were randomly divided into the treatment and control groups according to the order of their diagnosis times. The patients in the treatment group received daily oral diclofenac sodium $\left(\right.$ Difene $^{\circledR}$ ) and kinesitherapy. The patients in the control group received only daily oral diclofenac sodium. The treatment group contained 50 patients (20 males and 30 females, aged 44-62 years, mean 53.0 \pm 5.6 ). The numbers of patients having disease durations of $<3$ months, 3 months to 1 year, $1-2$ years and 2-3 years were 9, 13, 18 and 10 , respectively. The control group contained 50 patients (18 males and 32 females, aged 41-63 years, mean 52.3 \pm 7.0 ). The numbers of patients having disease durations of $<3$ months, 3 months to 1 year, $1-2$ years and 2-3 years were 8, 15, 20 and 7, respectively. The patients in the two groups were comparable in gender distribution, age and disease duration without any statistically significant differences $(\mathrm{P}<0.05)$. The study was approved by the ethics committee of Yancheng Health Vocational and Technical College, Yancheng, China. 
Diagnostic criteria. The knee osteoarthritis diagnostic criteria were as defined by the American Rheumatism Association (4): a) knee joint disease (pain duration $\geq 14$ days within one month prior to treatment); b) X-ray showing osteophyte formation at the joint margins; c) clear, sticky synovial fluid with a leukocyte count $<2,000$ cells $/ \mathrm{ml}$; d) absence of synovial fluid index, age $>40$ years; e) knee morning stiffness $<30 \mathrm{~min}$; and f) crepitation during knee motion. Patients with a,b, or a,c,e,f, or a,d,e,f combinations of the above criteria were confirmed.

Inclusion and exclusion criteria. Inclusion criteria included: diagnostic criteria of KOA; not receiving other treatment methods or medication; age 40-70 years; and a disease classification of I-III by X-ray. Exclusion criteria included: significant stenosis of the joint space or bone bridge connection between the joints manifested as bony ankylosis; disease classification IV by X-ray; primary disease of the knee affecting joint structures; active gastrointestinal diseases and/or esophageal or peptic ulcer within 30 days of the trial; concurrent cardiovascular, cerebrovascular, liver, kidney or hematopoietic system diseases; mental illness; pregnancy and lactation; and known allergy to diclofenac.

\section{Experimental methods}

Treatment methods. The patients in the control group received oral diclofenac sodium (75 $\mathrm{mg}$, twice daily). Patients in the treatment group received oral diclofenac sodium (75 mg, twice daily) and kinesitherapy. Kinesitherapy included knee joint flexion and extension (active exercise) followed by isometric contraction of the quadriceps femoris at angles of $0^{\circ}$ and $90^{\circ}$ from the knee joint. Contractions were held continuously for $10 \mathrm{sec}$ followed by $10 \mathrm{sec}$ of relaxation, repeated 10 times for one exercise circle. The patients also performed 5 circles at each angle, with 1 min between circles. Isometric exercises were performed in the sitting position to avoid the effects of weight-bearing on the knee joints. Patients carried out the exercises twice per day in the morning and afternoon, 4 days per week (5). The two groups received treatment for 4 consecutive weeks.

Functional evaluation of the knee joint. The joint function index refers to the sum of the evaluation scores for rest pain, activity pain, tenderness, swelling and joint function. Rest pain was scored as: painless; mild pain, no effect on life and work; moderate pain, effect on life and work; or severe pain, effect on sleep. The activity pain was scored as: painless; mild pain, pain during forced activity; moderate pain, pain during common activity; or severe pain, pain during light activity. Tenderness was scored as: no tenderness; mild tenderness, no effect on activity; moderate tenderness, frown; or severe tenderness, retreat. Swelling was scored as: no swelling; light swelling, limited to local sites of the joints; moderate swelling, in the joint range; or severe swelling, diffusing to the surrounding tissue. Joint function was scored as: normal; mild limitation, no effect on life and work; moderate limitation, effect on life and work; or severe limitation, sitting and/or lying position, unable to take care of themselves. For all parameters, the four possible scores were $0,1,2$ and 3 (6).

Outcome measures. Synovial fluid $(1.5 \mathrm{ml})$ was extracted from the medial compartment of the knee prior to and following 4 weeks of treatment and was stored at $-20^{\circ} \mathrm{C}$ until further

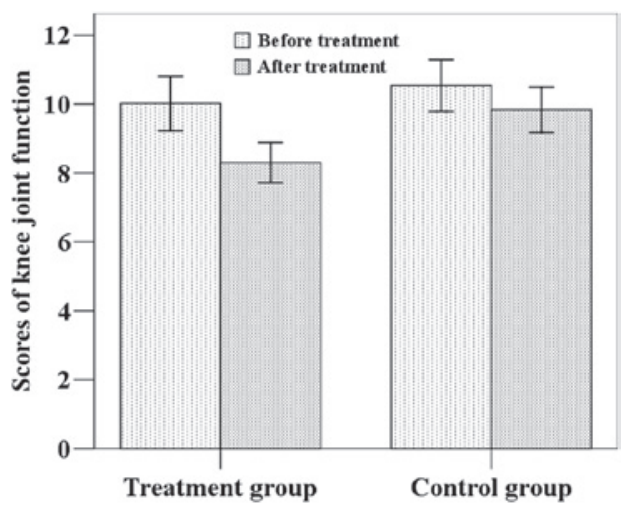

Figure 1. The index score (mean $\pm \mathrm{SD}$ ) of knee joint function for the two groups.

analysis. An enzyme-linked immunosorbent assay (ELISA) was used to evaluate the TNF- $\alpha$, hs-CRP and MMP-3 levels in the synovial fluid. The ELISA kits were acquired from Wuhan Boster Biological Engineering, Ltd. (Wuhan, China). The assays were performed according to the manufacturer's instructions.

Therapeutic effect criteria. The therapeutic effect was determined using the Clinical Guideline of New Drugs for Traditional Chinese Medicine (7). The therapeutic effects were divided into four grades: clinically-controlled; significantly effective; effective; and ineffective.

Statistical analysis. SPSS 17.0 software was used for analysis. The independent Student's t-test was used to compare the ages of the two groups and the $\chi^{2}$ test was used to compare gender and disease duration difference. Efficacies were compared using the Mann-Whitney U test. The knee function index scores and cytokine levels prior to and following treatment were compared by repeat-measures analysis of variance. The above analyses were two-sided and $\mathrm{P}<0.05$ was considered to indicate a statistically significant result.

\section{Results}

Knee function index scores. The mean knee function index scores $( \pm \mathrm{SD})$ of the patients in the treatment group were 10.0 $( \pm 2.8)$ at baseline and $8.3( \pm 2.1)$ following treatment, while the scores of the patients in the control group were $10.5( \pm 2.7)$ at baseline and $9.8( \pm 2.3)$ following the 4-week study (Fig. 1). Statistical analysis revealed that the post-treatment knee function index scores of the patients in the two groups were significantly lower than those before treatment $(\mathrm{P}<0.05)$. The score in the treatment group was significantly lower than that in the control group $(\mathrm{F}=4.695, \mathrm{P}=0.033)$.

TNF- $\alpha, h s-C R P$ and MMP-3 expression in synovial fluid. The mean $( \pm \mathrm{SD})$ TNF- $\alpha$, hs-CRP and MMP-3 levels in the treatment group were $0.88(0.26), 11.33(0.90)$ and 140.94 (22.02) $\mu \mathrm{g} / \mathrm{ml}$ at baseline and $0.50(0.21), 6.01(1.13)$ and 102.51 (21.84) $\mu \mathrm{g} / \mathrm{ml}$ following treatment. The mean $( \pm \mathrm{SD}) \mathrm{TNF}-\alpha$, hs-CRP and MMP-3 levels in the control group were 0.89 (0.26), $11.46(0.84)$ and $141.92(23.78) \mu \mathrm{g} / \mathrm{ml}$ at baseline and 
Table I. Comparison of the therapeutic efficacy between groups $[\mathrm{n}(\%)]$.

\begin{tabular}{|c|c|c|c|c|c|}
\hline Groups & Cases & $\begin{array}{l}\text { Clinically- } \\
\text { controlled }\end{array}$ & $\begin{array}{l}\text { significantly } \\
\text { effective }\end{array}$ & effective & ineffective \\
\hline Treatment group & 50 & $11(22.0)$ & $21(42.0)$ & $13(26.0)$ & $5(10.0)$ \\
\hline Control group & 50 & $3(6.0)$ & $16(32.0)$ & $23(46.0)$ & $8(16.0)$ \\
\hline Total & 100 & $14(14.0)$ & $37(37.0)$ & $36(36.0)$ & $13(13.0)$ \\
\hline
\end{tabular}

$\mathrm{Z}=2.736, \mathrm{P}=0.006$.

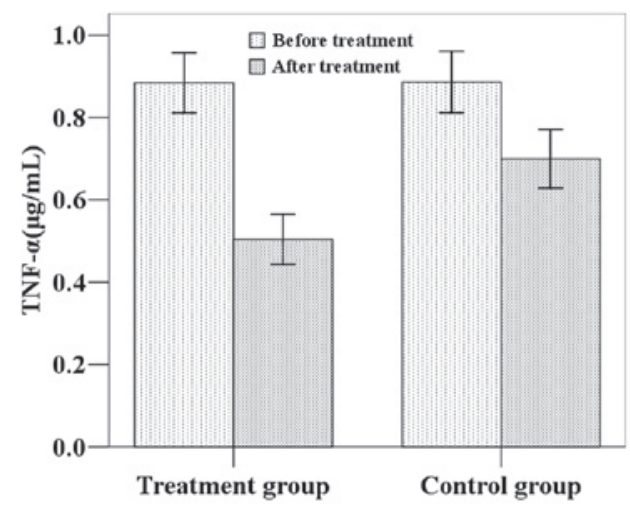

Figure 2. Tumor necrosis factor- $\alpha(\mathrm{TNF}-\alpha)$ levels $($ mean $\pm \mathrm{SD})$ in the joint synovial fluid of patients from the two groups.

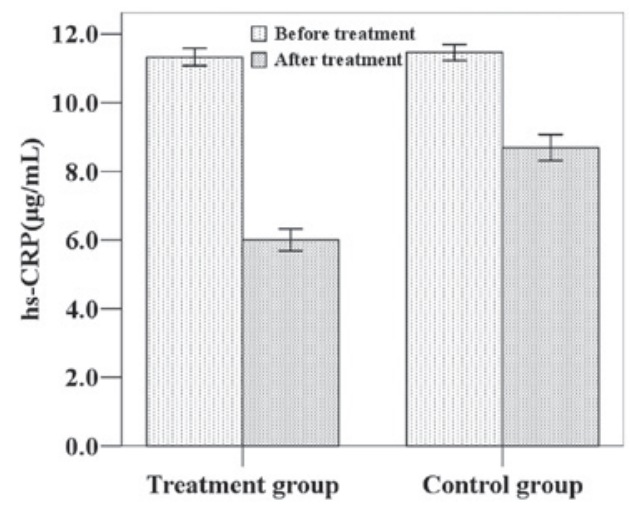

Figure 3. High sensitivity C-reactive protein (hs-CRP) levels (mean $\pm \mathrm{SD}$ ) in the joint synovial fluid of patients from the two groups.

$0.70(0.25), 8.69$ (1.36) and $120.06(21.53) \mu \mathrm{g} / \mathrm{ml}$ following the four-week study period (Figs. 2-4). Statistical analysis demonstrated that the post-treatment TNF- $\alpha$, hs-CRP and MMP-3 levels were significantly lower than the baseline values in the two groups. In addition, post-treatment TNF- $\alpha$, hs-CRP and MMP-3 levels in the treatment group were significantly lower than in the control group $(\mathrm{P}<0.05)$.

Clinical efficacy comparison. The efficacy rate was $90.0 \%$ (45/50) in the treatment group and 84\% (42/50) in the control group, as shown in Table I. Statistical analysis showed that the therapeutic effect in the kinesitherapy treatment group was

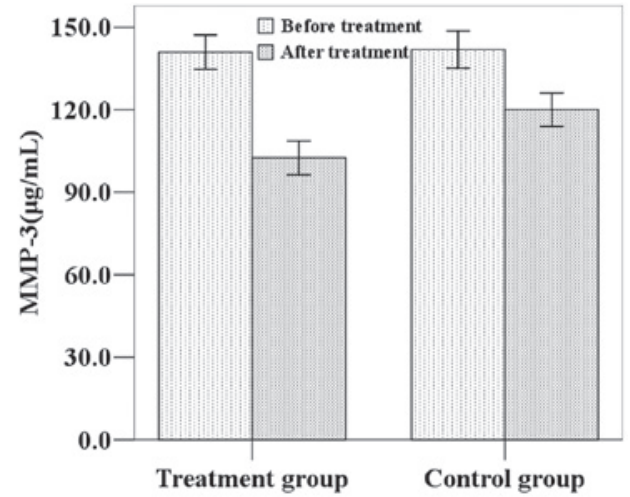

Figure 4. Matrix metalloproteinase-3 (MMP-3) levels (mean \pm SD) in the joint synovial fluid of patients from the two groups.

significantly improved compared with that in the control group $(\mathrm{Z}=2.736, \mathrm{P}=0.006)$.

\section{Discussion}

KOA primarily affects the physical functions and activities of patients, particularly daily activities related to walking and transferring that are essential for the maintainance of daily independence and the quality of life (8). Maly et al (9) found that the pain and limited activity of KOA reduces patients' evaluations of self-worth. Therefore, researchers continue to search for safe and reliable treatment methods to effectively treat KOA and reduce its adverse effects on the quality of life.

Kinesitherapy has previously been proposed as the treatment of choice for KOA in certain countries (10). In this study, kinesitherapy was used to treat KOA and its effects on knee function and the levels of TNF- $\alpha$, hs-CRP and MMP-3 in synovial fluid were observed. The results revealed a gradual improvement in knee function following treatment. The combination of kinesitherapy and diclofenac was able to improve joint function and movement and may support healthy cartilage metabolism and repair.

KOA pathogenesis is an inflammatory process involving multiple cytokines and inflammatory mediators (11). TNF- $\alpha$ selectively inhibits the generation of cartilage collagen and proteoglycan synthesis and promotes its degradation. Increased cytokine levels in the knee joint synovial fluid are closely related to KOA severity (12). hs-CRP is a highly sensitive marker of inflammation and Spector et al found that hs-CRP levels were significantly higher in the serum of KOA 
patients than that of healthy controls and that hs-CRP levels positively correlated with osteoarthritis disease activity (13). It is considered that the pathogenesis of knee osteoarthritis primarily involves the degeneration and degradation of articular cartilage, while the main cause of cartilage degeneration is the abnormal degradation of the extracellular matrix on which chondrocytes are dependent.

MMPs are involved in the degradation of the extracellular matrix. MMP-3 is an important member of the MMP family that is able to degrade extracellular proteoglycans, type IV collagen and a number of other matrix protein substrates. Type IV collagen is a non-indigenous fibrosis collagen primarily located at the articular cartilage surface. MMP-3 is also involved in the activation of interstitial collagenase. Interstitial collagenase degrades type II collagen and so MMP-3 is indirectly involved in type II collagen degradation. Studies have revealed that the serum and synovial fluid MMP-3 levels of patients with osteoarthritis are significantly higher than those of normal controls and are related to the severity of the joint disease (14). Therefore, detection of TNF- $\alpha$, hs-CRP and MMP-3 levels in the synovial fluid of KOA patients may be used for comprehensive diagnosis and for the evaluation of disease severity and prognosis. The results of this study show that kinesitherapy significantly reduces TNF- $\alpha$, hs-CRP and MMP-3 levels in the synovial fluid of KOA patients. This mechanistic effect may inhibit inflammatory damage to chondrocytes and the cartilage matrix, delay articular cartilage degeneration and improve the therapeutic effect and knee function.

\section{References}

1. Page CJ, Hinman RS and Bennell KL: Physiotherapy management of knee osteoarthritis (Review). Int J Rheum Dis 14: 145-151, 2011.

2. Topp R, Woolley S, Hornyak J III, Khuder S and Kahaleh B: The effect of dynamic versus isometric resistance training on pain and functioning among adults with osteoarthritis of the knee. Arch Phys Med Rehabil 83: 1187-1195, 2002.
3. Deyle GD, Allison SC, Matekel RL, Ryder MG, Stang JM, Gohdes DD, Hutton JP, Henderson NE and Garber MB: Physical therapy treatment effectiveness for osteoarthritis of the knee: a randomized comparison of supervised clinical exercise and manual therapy procedures versus a home exercise program. Phys Ther 85: 1301-1317, 2005.

4. Altman R, Asch E, Bloch D, et al: Development of criteria for the classification and reporting of osteoarthritis. Classification of osteoarthritis of the knee. Diagnostic and Therapeutic Criteria Committee of the American Rheumatism Association. Arthritis Rheum 29: 1039-1049, 1986.

5. Yang YH, Su GH, Luo JM, et al: Overall acupotome zonulolysis in combination of kinesitherapy in treatment of 44 cases of knee osteoarthritis. Academic Journal of Anhui University of Traditional Chinese Medicine 28: 43-45, 2009 (In Chinese).

6. Kang XP and Ye YJ: The effect of articular injection of chitosan on knee function and inflammatory factors in synovial fluid. Chinese Clinical Rehabilitation 9: 42-43, 2008 (In Chinese).

7. Zheng XY (ed): Clinical Guideline of New Drugs for Traditional Chinese Medicine in Treatment of Osteoarthritis. In: Clinical Guideline of New Drugs for Traditional Chinese Medicine. China Medical Science and Technology Press, Beijing, pp350-353: 2002 (In Chinese)

8. Kuptniratsaikul V, Tosayanonda O, Nilganuwong $\mathrm{S}$ and Thamalikitkul V: The efficacy of a muscle exercise program to improve functional performance of the knee in patients with osteoarthritis. J Med Assoc Thai 85: 33-40, 2002.

9. Maly MR and Krupa T: Personal experience of living with knee osteoarthritis among older adults. Disabil Rehabil 29: 1423-1433, 2007.

10. O'Reilly SC, Muir KR and Doherty M: Effectiveness of home exercise on pain and disability from osteoarthritis of the knee: a randomised controlled trial. Ann Rheum Dis 58: 15-19, 1999.

11. Lin MN and Liu XX: Synergistic effect of cytokines in osteoarthritis. Academic Journal of Fujian College of Traditional Chinese Medicine 16: 69-70, 2006 (In Chinese).

12. Wang DH, Bao F, Wu ZH, Sun $\mathrm{H}$ and Zhang YX: Influence of acupuncture on IL-1beta and TNF-alpha expression in the cartilage of rats with knee osteoarthritis. Zhongguo Gu Shang 24: 775-778, 2011 (In Chinese).

13. Spector TD, Hart DJ, Nandra D, Doyle DV, Mackillop N, Gallimore JR and Pepys MD: Low-level increases in serum C-reactive protein are present in early osteoarthritis of the knee and predict progressive disease. Arthritis Rheum 40: 723-727, 1997.

14. Aktas E, Sener E and Gocun PU: Mechanically induced experimental knee osteoarthritis benefits from anti-inflammatory and immunomodulatory properties of simvastatin via inhibition of matrix metalloproteinase-3. J Orthop Traumatol 12: 145-151, 2011. 